This is an Accepted Manuscript of an article published by Taylor \& Francis in 'Archives of Suicide Research' on 2014-07-24, available online: https://www.tandfonline.com/10.1080/13811118.2014.915778.

\title{
Non-Suicidal Self-Injury among Adolescents in Amman, Jordan
}

While previous research has demonstrated cross-national differences in non-suicidal self-injury (NSSI), most studies to date have taken place in North America. The present study investigated the prevalence and characteristics of NSSI in a sample of 952 Jordanian adolescents (49.8\% female) between the ages of 11-19 years. Participants completed a screening measure to assess occurrence of NSSI and its characteristics. Results indicate an overall lifetime prevalence of $22.6 \%(n=215)$, with significantly more males $(26.98 \%, n=129)$ than females $(18.14 \%, n=86)$ reporting having engaged in NSSI at least once in their lifetime. This study provides empirical evidence that adolescent engagement in NSSI occurs at similar prevalence levels in Jordan, relative to North American samples, whereas gender comparisons of prevalence and characteristics revealed several differences.

Keywords adolescents, culture, Jordan, non-suicidal self-injury (NSSI), self-harm, selfinjuring behavior

Non-suicidal self-injury (NSSI) is defined as " purposely inflicting injury that results in immediate tissue damage, done without suicidal intent, and not socially sanctioned", (Nixon \& Heath, 2008a, p. 4). Although research in the area of NSSI in community samples is ever growing, it has been mainly limited to North American, European, and Australian samples (e.g., Hawton, Harriss, Hall et al., 2003; Heilbron \& Prinstein, 2010; Lloyd-Richardson, Perrine, Dierker et al., 2007; Ross \& Heath, 2002; Williams \& Hasking, 2009). There are few studies examining NSSI outside of these populations, however, there are some studies of self-harm, which has related but unique behavioral features. Thus, there is little information on NSSI beyond the findings from these westernized countries. The present study extends this literature by examining NSSI for 
the first time in a school-based sample of adolescents in the Middle East.

\section{Prevalence of NSSI}

The majority of community-based studies show that between $14 \%$ and $24 \%$ of North American adolescents report having engaged in NSSI at least once in their lifetime (e.g., Hankin \& Abela, 2011; Heath, Schaub, Holly et al., 2008; Jacobson \& Gould, 2007; Laye-Gindhu \& Schonert- Reichl, 2005; Muehlenkamp \& Gutierrez, 2007; Nock, 2009; Ross \& Heath, 2002).

Outside of North America, studies show that the prevalence of NSSI is variable, ranging from $5.5 \%$ to $65.9 \%$ for lifetime prevalence in adolescent samples. Specifically, based on single studies, the estimated lifetime prevalence rate of NSSI among an adolescent population was $5.5 \%$ in Hungary (Csorba, Szelesne, Steiner et al., 2005), 13.8\% in Scotland (O’Connor, Rasmussen, Miles et al., 2009), 21.4\% in Turkey (Zoroglu, Tuzun, Sar et al., 2003), 25.6\% in Germany (Plener, Libal, Keller et al., 2009), $\quad 30.7 \%$ in Belgium (Muehlenkamp, Williams, Gutierrez et al., 2009), and a higher lifetime prevalence rate of $65.9 \%$ reported in Sweden (Lundh, Karim, \& Quilisch, 2007). In a study of NSSI, $12.5 \%$ of Sami and Norwegian adolescents reported engaging in NSSI in the last 6 months (Kvernmo \& Rosenvinge, 2009). Thus, there is substantial variability in the reported prevalence of NSSI across countries, with Hungary appearing to have lower prevalence rates while other countries, with the exception of Sweden, report prevalence rates similar to North America.

Although socio-cultural factors may play a fundamental role in accounting for variations between reported NSSI prevalence rates across countries, it is imperative to take into account methodological factors. The low prevalence rates observed in Hungary, and the 
very high prevalence rates reported in Sweden may be accounted for by the diverse ways in which researchers questioned participants about NSSI behavior. Specifically, the study of Hungarian adolescents (Csorba, Szelesne, Steiner et al., 2005) assessed the occurrence of NSSI using a translated version of the Ottawa Self-Injury Inventory (Cloutier \& Nixon, 2003) that examines more severe forms of self-injury, which are less frequent. Conversely, the study conducted in Sweden utilized a simplified version of the Deliberate Self-harm Inventory (Gratz, 2001), which assesses a milder and more common range of self-injurious behaviors.

In a unique cross-national study of NSSI, Plener, Fischer, In-Albon et al. (2013) assessed and compared prevalence rates of NSSI among 1,339 adolescents from Austria, Germany, and Switzerland. Results indicate that $18.8 \%(n=252)$ of the participants reported having injured themselves within the last 6 months. There were significant differences in the NSSI 6-month prevalence rates across countries, with highest rates reported in Germany and lowest in Switzerland (14.6\% and 7.6\%, respectively). These findings reflect the variability in NSSI rates across neigh- boring countries, and further support the assumption that NSSI among adolescents varies across cultures.

\section{Characteristics of NSSI: Frequency, Onset, and Methods}

With regard to frequency, or the number of times an individual has engaged in NSSI, there appears to be great variation (e.g., Laye-Gindhu \& Schonert-Reichl, 2005; Muehlenkamp \& Gutierrez, 2007; Ross \& Heath, 2002). Among community samples of adolescents from North American, Australian, and European samples, there is variability in lifetime fre- quency of NSSI ranging from a single incident to hundreds of incidents (e.g., Hawton, Harriss, Hall et al., 2003; Jacobson \& Gould, 2007; Martin, Swannell, 
Hazell et al., 2010; Nock, 2010). However, no cross-national comparison of the frequency of NSSI among adolescent community samples exist and, thus, our understanding of how this may differ across cultures remains limited.

Regarding the age of onset of NSSI, retrospective reports in community populations indicate that engagement in NSSI commonly begins between the ages of 11 to 15 years (Hankin \& Abela, 2011; Heath, Schaub, Holly et al., 2008; Jacobson \& Gould, 2007; Muehlenkamp \& Gutierrez, 2007; Nixon, Cloutier, \& Aggarwal, 2002; Nixon \& Heath, 2008b; Nock \& Prinstein, 2005). In a cross-sectional survey of a representative sample of 12,006 Australians from randomly selected households, which included 1,475 adolescents (12.3\%, aged 10-17 years), Martin and colleagues (2010) reported that selfinjury peaked between the ages of 15 and 24 years in females, and between the ages of 10 and 19 years in males. This suggests a possi- bility of an earlier age of onset for males compared to females in this sample, and indicates that various aspects may influence age of onset, such as gender or geographical region.

Although NSSI is most commonly associated with skin cutting, it encompasses behaviors including (but not limited to) burning, scratching, picking or interfering with wound healing, and punching oneself to cause bruising (e.g., Lloyd-Richardson, Perrine, Dierker et al., 2007; Nock, 2010; Ross \& Heath, 2002). In North American, Australian, German, and Turkish com- munity samples, the most commonly reported methods of NSSI are cutting and scratching, with self-punching and hitting less common (Hasking, Momeni, Swannell et al., 2008; Heath, Toste, Nedecheva et al., 2008; Laye-Gindhu \& SchonertReichl, 2005; Martin, Swannell, Hazell et al., 2010; Nixon, Cloutier, \& Jansson, 2008; Plener, Libal, Keller et al., 2009; Zoroglu, Tuzun, Sar et al., 2003). Plener and colleagues 
This is an Accepted Manuscript of an article published by Taylor \& Francis in 'Archives of Suicide Research' on 2014-07-24, available online: https://www.tandfonline.com/10.1080/13811118.2014.915778.

(2009), in his first cross-national comparison of NSSI found that, while cutting was significantly more common in the United States than in Germany, there were no differences in the use of scratching and hitting oneself.

\section{Gender and Age Difference in NSSI}

Studies examining gender differences in the prevalence rate of NSSI within com- munity samples of adolescents have been inconsistent. Some studies have found a higher prevalence of NSSI among females (e.g., Laye-Gindhu \& Schonert-Reichl, 2005; Muehlenkamp \& Gutierrez, 2007; Muehlenkamp, Williams, Gutierrez et al., 2009; Nixon, Cloutier, \& Jansson et al., 2008; Plener, Libal, Keller et al., 2009; Ross \& Heath, 2002; Sornberger, Heath, Toste et al., 2012; Yates, Tracy, \& Luthar, 2008), while others have found similar rates between female and male adolescents (e.g., Claes, Houben, Vandereycken et al., 2010; Richardson, Perrine, Dierker et al., 2007; Muehlenkamp \& Gutierrez, 2004; Zoroglu, Tuzun, Sar et al., 2003). In summary, research on gender differences in NSSI yields conflicting findings. Variability seems to arise, and may be accounted for by methods of data collection, operational definitions of NSSI, and sample sizes. Specifically, studies that employ measures that include a broader range of behaviors that are outside the scope of NSSI include suicidal behaviors in their definitions, and use smaller sample sizes may have yielded gender differences in NSSI (Claes, Houben, Vandereychen et al., 2010).

Beyond prevalence rates, research on gender differences in other characteristics of NSSI remains limited. In a recent study examining gender differences in preva- lence, method, and location of NSSI among a sample of 7,126 adolescents (50.8\% female); results revealed a detailed pattern whereby female and male adolescents differed across these 
variables (Sornberger, Heath, Toste et al., 2012). Specifically, females reported higher rates of NSSI and were significantly more likely than males to cut and scratch themselves; on the other hand, males were more likely than females to burn themselves, bang their heads, and punch themselves. Results for the locations of NSSI also indicated gender differences with females reporting more injuries to arms and legs than their male counterparts, while males reported more injuries to the chest, face, and genitals. This pattern suggests that adolescent males who self-injure may be at a greater medical risk than their female counterparts (Sornberger, Heath, Toste et al., 2012).

Although gender differences have been reported in the prevalence of NSSI in adolescence (e.g., Laye-Gindhu \& Schonert-Reichl, 2005; Muehlenkamp, Williams, Gutierrez et al., 2009; Nixon, Cloutier, \& Jansson et al., 2008), such findings have not been seen in young adults (Sornberger, Heath, Toste et al., 2012) indicating that gender differences in NSSI may vary by age. In a recent study, Barrocas, Hankin, Young et al. (2012) assessed the grade by gender interaction for NSSI among 665 children and adolescents (55\% female), between the ages of 7 and 16 years. Results indicated a significant interaction effect of grade by gender for NSSI engagement such that there was no gender difference in lifetime reports of NSSI in grades three or six but females did report more NSSI in grade nine than their male peers. These findings suggest that gender differences in NSSI may occur with the onset of adolescence, although these results are preliminary and require further study.

In another study of NSSI among 1,931 underserved middle and high school students (51.4\% female), Gratz and colleagues (2012) found that rates of NSSI vary as a function of the interaction of gender, racial=ethnic background, and school-level. In particular, 
This is an Accepted Manuscript of an article published by Taylor \& Francis in 'Archives of Suicide Research' on 2014-07-24, available online: https://www.tandfonline.com/10.1080/13811118.2014.915778.

results show that African American boys, particularly in middle school, reported higher rates of DSH behaviors than other participants. There- fore, these findings suggest that the etiology and correlates of this behavior may further differ across specific subsets of adolescents.

\section{Cross-Cultural Studies of Self-Harm}

While few cross-cultural studies exam- ining NSSI exist, there are several studies that examine "self-harm" across diverse cultures among adolescents and adults both community and inpatient samples (Abu Madini \& Rahim, 2001; Kinyanda, Hjelmeland, \& Musisi et al, 2005; Parkar, Dawani, \& Weiss, 2006; 2008). Although NSSI and selfharm have some shared features, they are quite distinct in that self-harm includes a broader range of self- injury, such as overdosing and jumping from heights (e.g., De Leo

\& Heller, 2004; Gratz, 2001; Hawton, Fagg, Simkin et al., 2000; Hawton, Harriss, Hall et al., 2003). As these behaviors have a high degree of overlap with suicidality, caution must be used when interpreting the literature on self-harm and relating these findings to NSSI, particularly within an international context as findings related to the prevalence, characteristics, and functions of self-harm are not necessarily pertinent to the study of NSSI.

In a cross-national study that specifically examined self-harm, Madge and colleagues (2008) compared self-harm behaviors among a community sample of 30,477 adolescents (51.3\% male) in seven countries: Belgium, England, Hungary, Ireland, the Netherlands, Norway, and Australia. Results indicated that the highest rates of reported lifetime selfharm behaviors were obtained in Belgium (6.5\%), followed by Norway (5.0\%), and England (4.8\%), while the Netherlands had the lowest rates (2.4\%). Similarly, Portzky, 
De Wilde, and Van Heeringen (2008) reported that rates of self-harm differ significantly between neighboring countries, where the lifetime prevalence rate of self-harm among Belgian and Dutch adolescents was $10.4 \%$ and $4.1 \%$ respectively. These rates are surprisingly low and may reflect the study's emphasis on suicidal behaviors.

Furthermore, these findings highlight a marked difference in the prevalence of self-harm when investigated cross- nationally. Given that self-harm includes self-injuring behaviors regardless of suicidal intent, it remains unclear whether these differences would also be observed with NSSI.

In the above study, Madge and colleagues (2008) reported that females had significantly higher rates of lifetime prevalence compared to males. Specifically, $13.5 \%$ of females and $4.3 \%$ of males reported a lifetime prevalence of self-harm. The higher rates in females may be due to the inclusion of overdose in self-harm which is known to occur more frequently among females (Rodham \& Hawton, 2009).

\section{Objectives of the Present Study}

Overall, research indicates that there are cross-national differences in NSSI with regard to prevalence and characteristics (e.g., frequency, methods, age of onset, gender). While most studies on NSSI to date have taken place in westernized coun- tries, no studies have been conducted in the Middle East. The present study had two central objectives related to the investigation of NSSI among a sample of adolescents in Amman, Jordan. We sought to: (a) examine the lifetime prevalence rates and characteristics of NSSI (e.g., frequency, duration of the behavior, age of onset, methods of injury), and (b) explore whether gender differences exist in the prevalence and characteristics of NSSI, as well as potential interactions with grade-level. 
This is an Accepted Manuscript of an article published by Taylor \& Francis in 'Archives of Suicide Research' on 2014-07-24, available online: https://www.tandfonline.com/10.1080/13811118.2014.915778.

\section{Method}

\section{Participants}

Data were collected from a sample of 966 adolescents enrolled in grades seven through twelve from five private schools in Amman, Jordan. Participants that did not complete the full survey, including the question related to NSSI engagement, were removed $(n=$ 14). The final sample of 952 students consisted of 474 females (49.8\%) and 478 males $(50.2 \%)$, ranging

in age from 11 to 19 years $(M=14.53, S D 1.71)$. The majority of participants were born in Jordan $(80.6 \%)$, while $19.4 \%$ of participants were born in other countries. Language spoken at home was reported as Arabic (55.8\%), both Arabic and English (37.2\%), English only (5.6\%),

and other $(1.4 \%)$.

\section{Measures}

The measure, in addition to all school and consent materials, was back translated into Arabic, the official language in Jordan. Translation was done under the super- vision of the research collaborator in Amman, Jordan in order to ensure the validity of the measure. All participants in this study were given the choice of completing the questionnaire in English or in Arabic. Almost half (48.3\%) chose to answer the questionnaire in English, while $51.7 \%$ of participants answered in Arabic. We were able to ascertain that all students had a basic level of English proficiency, as one of the primary languages of education and assessment in these schools. Potential language effects were considered and no statistically significant differences were found in the rates of NSSI (or other variables of interest) between participants who completed the English versus Arabic 
version of the measure.

Participants in this study completed an adapted version of the How I Deal with Stress questionnaire (HIDS; Ross \& Heath, 2007), translated into Arabic. The HIDS is a 29item self-report questionnaire, designed to screen for NSSI by assessing for several adaptive and maladaptive coping strategies that may be employed by adolescents. The HIDS measure was adapted for ethical and cultural considerations. Specifically, questions addressing alcohol use and sex were excluded from the HIDS. Participants were asked to rate their use of each strategy to deal with stress on a 4-point Likert scale: never, sometimes, few times, or frequently. One of the listed strategies was " physically hurt myself on purpose.' Participants were asked to complete a follow-up section on their self-injury if they had indicated ever using this strategy.

The follow-up questions asked participants to indicate method of self-injury without suicidal intent by choosing from a list of methods (cutting, burning, scratching, banging, or punching) with the option of specifying any methods not listed distinguishing NSSI from other potentially harmful behaviors. The total number of endorsed methods was calculated for each participant. Participants were also asked to indicate age of first episode of self-injury and how many times they had hurt them- selves on purpose throughout their life (one time, 2-4 times, 5-10 times, 11-50 times, 51-100 times, or more than 100 times). Participants were further questioned about their current NSSI status with the option of indicating that they: (a) had permanently stopped engaging in NSSI; (b) were currently engaging in NSSI; or, (c) were unsure as to whether they are currently engaging in the behavior or if they have stopped.

The original version of the HIDS, in English, has been used extensively in com- munity 
samples of youth and young adults (e.g., Heath, Schaub, Holly et al., 2008; Heath, Toste, Nedecheva et al., 2008; Sornberger, Heath, Toste et al., 2012). The psychometric properties of the HIDS as a screening measure for NSSI have been supported by past analyses. The HIDS has been found to have a reported Cronbach's alpha of 0.78 , suggesting reasonable internal consistency (Heath, Toste, Nedecheva et al., 2009); additionally, construct validity of this measure has been supported through multidimensional scaling analysis (Toste, Grouzet, Heath et al., 2006, Toste, Heath, Grouzet el al., 2013). The Cronbach's alpha for the present sample was 0.79, suggesting reasonable internal consistency. Further, the NSSI item on the HIDS has high test-retest reliability, $r$ (226) .84, at six months (Toste, Christie, \& Heath, 2011).

\section{Procedure}

Participants were recruited to complete a survey on coping and stress in youth. Parental consent was sought for all students in grades seven through twelve at participating schools. Students who received parental consent were asked to provide their own assent prior to completing the questionnaire. The participation rate for this study was $86.3 \%$. The measure was administered in a group setting; students had 30 minutes to complete the questionnaire individually during regular class time. Teachers provided students who did not receive parental consent, or did not provide assent, with work to complete during the 30-minute session.

\section{Results}

\section{Reported Prevalence and Characteristics of NSSI}

The rate of NSSI occurrence within this sample was calculated using the responses to the follow-up question “'have you intentionally hurt yourself without suicidal intent?'”. From 
the total sample of adolescents ( $\mathrm{n} 952$ ), 22.6\% (n 215) of participants indicated that they had hurt themselves on purpose, without suicidal intent, at least once in their lifetime. Table 1 provides the rates of NSSI for the sample of adolescents who reported engaging in NSSI broken down by grade. Figure 1 provides the lifetime frequency for 205 respondents (10 participants did not complete the follow-up section).

When reporting the characteristics of NSSI within this sample, information was not always available from all 215 participants who reported having engaged in NSSI as some participants chose to skip questions in the follow-up section. Thus, the number of participants is specified in text for each question. When asked about the participants' last incident of NSSI, $80.1 \%$ (136 of 170 participants) indicated that they had last engaged in NSSI within the last year, $11.6 \%(n=20)$ indicated in the last three years, $4.2 \%(n=7)$ indicated more than three years ago, while $4.1 \%(n=7)$ reported that they could not remember when they last engaged in NSSI. When asked about their current NSSI status, $28.4 \%$ ( $n=60$ of 211 participants) indicated that they have permanently stopped engaging in NSSI, $21.8 \%(n=46)$ reported currently engaged in NSSI, while $49.8 \%(n=$ 105) responded with "maybe or I don't know' regarding the con- tinued use of NSSI.

Among 180 participants, the reported mean age of onset was 11.11 years $(S D=3.06)$. The distribution of reported age when participants first engaged in NSSI is presented in Figure 2. Reported methods of engaging in NSSI were as follows: scratching $(29.7 \%, 62$ of 204 participants), skin cutting $(28.7 \%, n=60)$, punching self $(28.2 \%, n=59)$, banging head $(24.4 \%, n=51)$, and burning $(9.6 \%, n=20)$. In addition, $12.7 \%(n=$ 26) of the sample reported engaging in an "other" method of NSSI such as: punching=banging objects or pulling out body hair. Overall, $78.4 \%(n=160)$ of 
participants reported having engaged in only one method of NSSI, $12.7 \%(n=26)$

reported two methods of NSSI, $6.4 \%(n=13)$ reported three methods of NSSI, and $2.5 \%(n=5)$ reported having engaged in four or more methods. Specifically, $18.14 \%(\mathrm{n}$ 86) of the female participants reported engaging in NSSI, while $26.98 \%(n=129)$ of the male

participants reported engaging in NSSI.

\section{Gender and Grade Differences}

To explore possible gender differences related to the prevalence, frequency, age of onset, and method of NSSI, chi-square analyses were conducted to compare responses between male and female participants. Results indicate that males reported significantly higher rates of NSSI than females, v2 $(1)=10.61, p<0.05, \mathrm{U}=0.105$. Specifically, $18.14 \%(n=$ 86) of the female participants reported engaging in NSSI, while $26.98 \%(n=129)$ of the male participants reported engaging in NSSI.

When examining frequency of NSSI for the full sample, no significant differences were found between male and female participants (see Table 2). In terms of age of onset for NSSI behavior, males were more likely than their female counterparts to engage in NSSI at age $10(\mathrm{v} 2(1)=4.221, p<0.05, \mathrm{U}=0.151)$ and younger $(\mathrm{v} 2(1)=4.245, p<0.05, \mathrm{U}=$ 0.151), although no gender differences were found at older ages (see Table 2).

A chi-square analysis was also conducted to further examine gender differences in the methods of NSSI endorsed by females and males (see Table 2). Results revealed that females were more likely than males to engage in cutting, v2(1) $6.668, p<0.05, \mathrm{U}=$ 0.179 , and scratching, v2(1) $14.768, p<0.01, \mathrm{U}=0.266$. Conversely, males were more likely than females to report self-punching, v2(1) $=10.301, p<0.05, \mathrm{U}=0.222$. No 
significant gender differences were found for other methods.

Binary logistic regression analyses were conducted to test grade by gender interaction in relation to a higher likelihood of engagement in NSSI. Results revealed a significant interaction effect of grade by gender $\left(\beta=0.743, p=0.017, X^{2}=3.38\right.$, odds ratio 2.103, $95 \%$ CI $[0.07,0.77])$ on engagement in NSSI. Follow-up chi square analyses indicated that males in grade seven reported significantly higher rates of NSSI engagement than their female counterparts $(\mathrm{v} 2(1)=10.782, p=0.001, \mathrm{~V}=0.262)$.

Thus, the prevalence of NSSI was higher for males in the early grades whereas females had a largely consistent prevalence rate in every grade. Figure 3 presents NSSI prevalence for female and male participants across grades seven through twelve.

\section{Discussion}

Although there has been ample research examining the occurrence of NSSI among community samples of adolescents, there have been no previous studies conducted in the Middle East. While this study does not examine the impact of culture on NSSI, the present study sought to extend our understanding of NSSI by considering how the etiology and characteristics of this behavior may vary among contexts and specifically, in

this study, a school-based sample of adolescents in Amman, Jordan. The results also have significant implications for guiding the efforts in establishing culturally sensitive clinical practice and preventive measures.

The current findings reveal a number of similarities to the prevalence NSSI that has been reported elsewhere. An overall lifetime prevalence rate of $22.6 \%$ was reported among this sample of Jordanian adolescents, with 52.7\% participants who self-injured reporting that they had physi- cally hurt themselves on purpose only one time, and $9.8 \%$ indicating that 
they hurt themselves over 100 times. This is similar to the prevalence rates ranging from $14 \%$ to $24 \%$ that have been reported in previous studies (e.g., Hankin \& Abela, 2011; Heath, Toste, Nedecheva, \& Charlebois, 2008; Jacobson \& Gould, 2007).

Although the prevalence rate of NSSI among female adolescents in this sample was in line with previous findings, the prevalence rate among males is inconsistent with previous research (e.g., Madge, Hewitt, Hawton et al., 2008; Muehlenkamp \& Guttierrez, 2007; Ross \& Heath, 2002; Sornberger, Heath, Toste et al., 2012). It remains unclear whether this gender difference observed in the reported lifetime prevalence may reflect a differential use of NSSI for Jordanian male and female adolescents. This noted pattern may be explained by social display rules that are gender specific regarding emotions across differing contexts (Polce-Lynch, Myers, Kilmartin et al., 1998). As such, female and male adolescents may follow differing emotional expressions that are compliant with display rules that are defined by gender and culture. An important contribution of the current study was the examination of NSSI across grades. The gender-by-grade interaction for lifetime prevalence revealed that more males reported engaging in NSSI in seventh grade, whereas female prevalence was relatively constant across grade-levels. Similar to the aforementioned gender difference, the high prevalence of males in grade seven reporting NSSI is not consistent with previous findings in North American contexts. Hilt and colleagues (2008) found no gender or grade differences in reports of NSSI among sixth, seventh, and eight grade students. Barrocas and colleagues (2012) did, however, report a grade-by-gender interaction in NSSI prevalence across third, sixth, and ninth grades, with no gender difference in early grades, but females in grade nine reporting higher prevalence than their male peers. The unusually high prevalence of NSSI in 
seventh grade males may be attributed to recall bias, where older adolescents may not recall their behavior to be severe enough to report it. This finding requires replication in order to understand if it is consistent among samples within this con- text, or if it may be a unique cohort effect. If replicated, the increased prevalence of NSSI amongst young males may reflect a differing course of behavior in Jordan. In addition to prevalence information, participants were asked about their NSSI engagement status, with $21.8 \%$ indicating that they were currently engaging in NSSI while almost half said that they "did not know" regarding the continued use of NSSI. While these statements are informative about the history of NSSI, they do not improve prediction about the likelihood of engaging in future NSSI behaviors (Janis \& Nock, 2008). That is to say, individuals may feel that they are certain about cessation or continuation of their NSSI, but this can change based on numerous factors. Thus, this question may not serve a great deal of utility in collecting information about adolescents' engagement in NSSI. Instead of relying on self-report measures, future research would benefit from using ecological momentary assessments, behavioral measures, and multimethod approach to improve the predictive power of future NSSI outcomes (e.g., Janis \& Nock, 2008; Rizvi \& Nock, 2008).

Although results indicate that age of onset for NSSI tends to be in early adolescence (11 to 13 years), a surprising number of Jordanian adolescents in this sample reported engaging in NSSI in preadolescence. Approximately $18.67 \%$ of females and $44 \%$ of males stated that they first self-injured at the age of 10 years or younger. Further, males were significantly more likely than their female counterparts to first engage in NSSI at a 
younger age. Therefore, the male participants in this sample seem to be more prone to early onset of NSSI when compared with females. Similar to previous research (Sornberger, Heath, Toste et al., 2012), this suggests that adolescent males may engage in more severe characteristics of NSSI which might put them at a greater risk than their female counterparts. Research shows that age of onset of NSSI has been reported to occur before puberty (Rodham \& Hawton, 2009; Ross \& Heath, 2002; Whitlock, Powers, \& Eckenrode, 2006). The current finding that nearly half of the males in this sample first self-injured in childhood may reflect a generalized difference in occurrence of NSSI among males, or it may be an indication of variations in this behavior amongst Middle Eastern sam- ples. Future research is necessary to investigate the possible contextual factors that contribute to a divergent male NSSI emergence. Furthermore research is needed to explore cultural and contextual differences in functions of NSSI which could potentially clarify the present gender differences. Consistent with other samples of adolescents who engage in NSSI (e.g., Ross \& Heath, 2002), the majority of Jordanian adolescents indicated they used only one method of NSSI (78.4\%). There were, however, some unexpected findings with regard to method. For Jordanian adolescents, there were comparable reports of scratching, cutting, and self-punching or head banging as the method of NSSI. These findings are in contrast to previous research results indicating cutting and=or scratching as a significantly more common method in most samples (Hasking, Momeni, Swannell et al., 2008; Martin, Swannell, Hazell et al., 2010; Nixon, Cloutier, \& Jansson et al., 2008). Further examination of these method differences with reference to the gender pattern revealed that female participants were significantly more likely than males to engage in cutting and scratching. Males, on the other hand, were 
significantly more likely than females to report punching them- selves. In light of the fact that in the present sample there was a higher prevalence of males than females reporting NSSI, the overall method results of comparable cutting, scratching, and punching may be a result of the greater number of males engaging in NSSI in the present sample. A second unexpected finding regarding method use was the absence of a gender difference regarding NSSI method of burning, which appears to be specific to this context. The majority of studies have found that burning is more commonly reported by males than females (e.g., Andover, Primack, Gibb et al., 2010; Martin, Swannell, Hazell et al., 2010; Sornberger, Heath, Toste et al., 2012). Although Zoroglu and colleagues (2003) in their study of Turkish adolescents also reported no gender difference in burning, the sample size was very small and the numbers endorsing burning were very low. It is also of particular interest to note that in a longitudinal study of 36 inpatient Jordanian adults, AlDabbas (2006) reports that deliberate self-burning was more commonly reported among females than males with a ratio of 6:1. While this study did not exclude self-injurious behaviors with suicidal intent, it suggests the use of burning as a form of self-injury may be more common in females in the Middle East than in other contexts.

\section{Limitations}

While the findings of this study con- tribute to our understanding of the presentation of NSSI within a Middle Eastern context, there are nonetheless certain limitations that should be noted. Specifically, the sample was drawn from a geographically distinct area of Jordan — specifically, five private high schools in Amman. Additionally, Amman is unique in regard to economic, ethnic, cultural, religious, levels of literacy, political, and other socio- demographic factors. As such, it is possible that the findings from this 
sample may not be generalizable to the larger population of adolescents in Jordan (or the Middle East) and, thus, further research is needed to investigate NSSI among a more representative sample. Another potential limitation is that there was no measure of participants' religion and intensity of religious beliefs. Since religion is an important aspect of Jordanian culture, such information would have been informative to the demographic description of the present sample and observed results.

The methodology of this study utilizes a self-report measure, which may have affected the validity of the results as it is subject to recall bias and social desirability (Nock \& Cha, 2009). While the prevalence of NSSI in this sample was established using the HIDS, this measure has not been validated in the population of interest. Furthermore, the psychometric properties of the HIDS-Arabic measure have not yet been examined, which may have influenced the observed rates of NSSI. Future research is needed to examine the reliability and validity of the HIDS in non-Western populations.

\section{Conclusion}

This study is the first to provide information related to the prevalence and characteristics of NSSI among adolescents in a sample from Amman, Jordan. There is a scarcity of research examining NSSI prevalence and characteristics among community samples of adolescents across cultures. This study provides empirical evidence that adolescent engagement in NSSI occurs in the Middle East at similar overall prevalence rates, but with a differing pattern of gender differences. These preliminary results contribute to our understanding of the importance of cultural differences in the occurrence and expression of NSSI and therefore demonstrate the need to extend our investigations of NSSI crossnationally. These findings add to the increasing findings that NSSI occurs across widely 
divergent contexts; however the characteristics of the behavior are not homogeneous. Thus, clinicians should be cognizant of the potential cultural variation of NSSI and not assume a uniformity of presentation and function. Future research is needed to confirm the present findings and examine the function of the behavior across genders. 
This is an Accepted Manuscript of an article published by Taylor \& Francis in 'Archives of Suicide Research' on 2014-07-24, available online: https://www.tandfonline.com/10.1080/13811118.2014.915778.

\section{References}

Abu Madini, M. S., \& Rahim, S. I. A. (2001).

Deliberate self-harm in a Saudi university hospital: A case series over six years (1994-2000). Arab Journal of Psychiatry, 12, 22-35.

Al-Dabbas, M. (2006). Deliberate self-burning: The psychosocial and clinical patterns among patients admitted to burn unit in King Hussein Medical Center, Jordan. Arab Journal of Psychiatry, 17, 33-39.

Andover, M. S., Primack, J. K., Gibb, B. E., \& Pepper, C. M. (2010). An examination of nonsuicidal self-injury in men: Do men differ from women in basic NSSI characteristics? Archives of Suicide Research, 14, 79-88. doi:10.1080=

13811110903479086

Barrocas, A. L., Hankin, B. L., Young, J. F., \& Abela,

J. R. (2012). Rates of non-suicidal self-injury in youth: Age, sex, and behavioral methods in a community sample. Pediatrics, 130, 130-139. doi:10.1542=peds.2011-2094

Claes, L., Houben, A., Vandereycken, W., \& Bijttebier, P. (2010). Brief report: The association between non-suicidal self-injury, self-concept and acquaintance with self-injurious peers in a sample of adolescents. Journal of Adolescence, 33, 775-778.

doi:10.1016=j.adolescence.2009.10.012

Cloutier, P. F., \& Nixon, M. K. (2003). The Ottawa Self-Injury Inventory: A preliminary evaluation. Abstracts to the 12th International Congress European Society for Child and Adolescent Psychiatry. European Child \& Adolescent Psychiatry, 12(Suppl. 1), 1-94.

Csorba, J., Szelesne, E. F., Steiner, P., Farkas, L., \& Nemeth, A. (2005). Symptom specificity of adolescents with self-injurious behavior. Psychiatria Hungarica, 20, 456-462. 
doi: 16479022

De Leo, D., \& Heller, T. S. (2004). Who are the kids who self-harm? An Australian self-report school survey. Medical Journal of Australia, 181, 140-144. doi:0025-729X

Gratz, K. L. (2001). Measurement of deliberate self-harm: Preliminary data on the deliberate self- harm inventory. Journal of Psychopathology and Behavioral Assessment, 23, 253263. doi:10.1023= A:1012779403943

Gratz, K. L., Latzman, R. D., Young, J., Heiden, L.

J., Damon, J. D., Hight, T. L., \& Tull, M. T. (2012). Deliberate self-harm among community adolescents in an underserved area: Exploring the moderating roles of gender, race, and school-level and association with borderline per- sonality features. Personality Disorders: Theory, Research, and Treatment, 3, 39-54. doi:10.1037= a0022107.

Hankin, B. L., \& Abela, J. R. (2011). Non-suicidal self-injury in adolescence: Prospective rates and risk factors in a 21/2 year longitudinal study. Psychiatry Research, 186, 65-70. doi:10.1016=j. psychres.2010.07.056

Hasking, P., Momeni, R., Swannell, S., \& Chia, S. (2008). The nature and extent of non-suicidal self-injury in a non-clinical sample of young adults. Archives of Suicide Research, 12, 208-218. doi: $10.1080=13811110802100957$

Hawton, K., Fagg, J., Simkin, S., Bale, E., \& Bond, A. (2000). Deliberate self-harm in adolescents in Oxford, 1985-1995. Journal of Adolescence, 23, 47-55. doi:10.1006=jado.1999.0290

Hawton, K., Harriss, L., Hall, S., Simkin, S., Bale, E., \& Bond, A. (2003). Deliberate self-harm in Oxford, 1990-2000: A time of change in patient characteristics. Psychological Medicine, 33, 987-95. doi:10.1017=S0033291703007943 
This is an Accepted Manuscript of an article published by Taylor \& Francis in 'Archives of Suicide Research' on 2014-07-24, available online: https://www.tandfonline.com/10.1080/13811118.2014.915778.

Heath, N. L., Schaub, K., Holly, S., \& Nixon, M. K. (2008). Self-injury today: Review of population and clinical studies in adolescents. In N. L. Heath \& M. K. Nixon (Eds.), Selfinjury in youth: The essen- tial guide to assessment and intervention (pp. 9-28). New York, NY: Routledge Press.

Heath, N. L., Toste, J. R., Nedecheva, T., \& Charlebois, A. (2008). An examination of nonsuicidal self-injury among college students. Journal of Mental Health Counseling, 30, 137-156. doi:1G1-178218778

Heilbron, N., \& Prinstein, M. J. (2010). Adolescent peer victimization, peer status, suicidal ideation, and non-suicidal self-injury: Examining concur- rent and longitudinal associations. Merrill-Palmer Quarterly, 56, 388-419. doi:10.1353=mpq.0.0049

Hilt, L. M., Nock, M. K., Lloyd-Richardson, E. E., \& Prinstein, M. J. (2008). Longitudinal study of non-suicidal self-injury among young adolescents: Rates, correlates, and preliminary test of an interpersonal model. The Journal of Early Adolescence, 28, 455-469. doi:10.1037=0022-006X.76.1.63

Jacobson, C., \& Gould, M. (2007). The epidemiology and phenomenology of non-suicidal selfinjurious behavior among adolescents: A critical review of the literature. Archives of Suicide Research, 11, 129-147. doi:10.1080=13811110701247602

Janis, I. B., \& Nock, M. K. (2008). Behavioral fore- casting does not improve the prediction of future behavior: A prospective study of self-injury. Journal of Clinical Psychology, 64, 1164-1174. doi:10.1002=jclp.20509

Kinyanda, E., Hjelmeland, H., \& Musisi, S. (2005). Negative life events associated with deliberate self-harm in an African population in Uganda. Crisis: The Journal of Crisis Intervention and Suicide Prevention, 26, 4-11. doi:10.1027=0227-5910. 
26.1.4

Kvernmo, S., \& Rosenvinge, J. H. (2009). Self-mutilation and suicidal behavior in Sami and Norwegian adolescents. International Journal of Circumpolar Health, 68, 235-248. doi: 19705656

Laye-Gindhu, A., \& Schonert-Reichl, K. A. (2005). Non-suicidal self-harm among community adolescents: Understanding the "whats" and "whys" of self-harm. Journal of Youth and Adolescence, 34, 447-457. doi:10.1007=s10964-005- 7262-Z

Lloyd-Richardson, E. E., Perrine, N., Dierker, L., \& Kelley, M. L. (2007). Characteristics and functions of non-suicidal self-injury in a community sample of adolescents. Psychological Medicine, 37, 1183. doi:10.1017=S003329170700027X

Lundh, L., Karim, J., \& Quilisch, E. (2007). Deliber- ate self-harm in 15-year-old adolescents: A pilot study with a modified version of the Deliberate Self-Harm Inventory. Scandinavian Journal of Psychology, 48, 33-41. doi:10.1111=j.1467-9450. 2007.00567.x

Madge, N., Hewitt, A., Hawton, K., Wilde, E. D., Corcoran, P., Fekete, S., .. . Ystgaard, M. (2008). Deliberate self-harm within an international com- munity sample of young people: Comparative findings from the Child \& Adolescent Self-harm in Europe (CASE) Study. Journal of Child Psychology

and Psychiatry, 49, 667-677. doi:10.1111=j.1469- 7610.2008.01879.x

Martin, G., Swannell, S., Hazell, P., Harrison, J., \& Taylor, A. (2010). Self-injury in Australia: A community survey. Medical Journal of Australia, 193, 506-510.

Muehlenkamp, J. J., \& Gutierrez, P. M. (2004). An investigation of differences between selfinjurious behavior and suicide attempts in a sample of adolescents. Suicide and LifeThreatening Behavior, 34, 12-23. doi:10.1521=suli.34.1.12.27769 
This is an Accepted Manuscript of an article published by Taylor \& Francis in 'Archives of Suicide Research' on 2014-07-24, available online: https://www.tandfonline.com/10.1080/13811118.2014.915778.

Muehlenkamp, J. J., \& Gutierrez, P. (2007). Risk for suicide attempts among adolescents who engage in non-suicidal self-injury. Archives of Suicide Research, 11, 69-82. doi: $10.1080=13811110600992902$

Muehlenkamp, J. J., Williams, K. L., Gutierrez, P. M., \& Claes, L. (2009). Rates of non-suicidal self- injury in high school students across five years. Archives of Suicide Research, 13, 317-239. doi: $10.1080=13811110903266368$

Nixon, M. K., Cloutier, P. F., \& Aggarwal, S. (2002). Affect regulation and addictive aspects of repeti- tive self-injury in hospitalized adolescents. Journal of the American Academy of Child \& Adolescent Psychiatry, 41, 1333-1341. doi:10.1097=00004583-

200211000-00015

Nixon, M. K., Cloutier, P., \& Jansson, S. M. (2008). Non-suicidal self-harm in youth: A population- based survey. Canadian Medical Association Journal, 178, 306-312. doi:10.1503=cmaj. 061693

Nixon, M. K., \& Heath, N. L. (2008a). Introduction to non-suicidal self-injury in adolescents. In M. K. Nixon \& N. L. Heath (Eds.), Self-injury in youth: The essential guide to assessment and intervention (pp. 1-16). New York, NY: Routledge.

Nixon, M., \& Heath, N. L. (2008b). Assessment of non-suicidal self-injury in youth. In M. K. Nixon \& N. L. Heath (Eds.), Self-injury in youth: The essential guide to assessment and intervention (pp. 1-6). New York, NY: Routledge Press.

Nock, M. K. (2009). Suicidal behavior among adoles- cents: Correlates, confounds, and (the search for) causal mechanisms. Journal of the American Academy of Child and Adolescent, 48, 237-239. doi:10.1097= CHI.0b013e318196b944

Nock, M. K. (2010). Self-injury. Annual Review of Clinical Psychology, 6, 339-363. 
doi:10.1146=annurev. clinpsy. 121208.131258

Nock, M. K., \& Cha, C. B. (2009). Psychological models of non-suicidal self-injury. In M. K. Nock (Ed.), Understanding non-suicidal self-injury: Origins, assessment, and treatment (pp. 65-78).

Washington, DC: American Psychological Association.

Nock, M. K., \& Prinstein, M. J. (2005). Contextual features and behavioral functions of selfmutilation among adolescents. Journal of Abnormal Psychology, 114, 140-146. doi:10.1037=0021-843X.114.1.140

O’Connor, R. C., Rasmussen, S., Miles, J., \& Hawton, K. (2009). Self-harm in adolescents: Selfreport survey in schools in Scotland. The British Journal of Psychiatry, 194, 68-72. doi:10. 1192=bjp.bp.107.047704

Parkar, S. R., Dawani, V., \& Weiss, M. G. (2006). Clinical diagnostic and sociocultural dimensions of deliberate self-harm in Mumbai, India. Suicide and Life-Threatening Behavior, 36, 223-238. doi:10.1521=suli.2006.36.2.223

Parkar, S. R., Dawani, V., \& Weiss, M. G. (2008). Gender, suicide, and the sociocultural context of deliberate self-harm in an urban general hospital in Mumbai, India. Culture, Medicine, and Psychiatry, 32, 492-515. doi:10.1007=s11013-008-9109-z

Plener, P. L., Fischer, C. J., In-Albon, T., Rollett, B., Nixon, M. K., Groschwitz, R. C., Schmid, M. (2013). Adolescent non-suicidal self-injury (NSSI) in German-speaking countries: Comparing preva- lence rates from three community samples. Social Psychiatry and Psychiatric Epidemiology, 48, 1439-

1445. doi:0.1007=s00127-012-0645-Z

Plener, P. L., Libal, G., Keller, F., Fegert, J. M., \& Muehlenkamp, J. J. (2009). An international 
This is an Accepted Manuscript of an article published by Taylor \& Francis in 'Archives of Suicide Research' on 2014-07-24, available online: https://www.tandfonline.com/10.1080/13811118.2014.915778.

com- parison of adolescent non-suicidal self-injury (NSSI) and suicide attempts:

Germany and the USA. Psychological Medicine, 39, 1549. doi:10.1017= S0033291708005114

Polce-Lynch, M., Myers, B. J., Kilmartin, C. T., Forssmann-Falck, R., \& Kliewer, W. (1998).

Gen- der and age patterns in emotional expression, body image, and self-esteem: A qualitative analysis. Sex Roles, 38, 1025-1048. doi:10.1023= A:1018830727244

Portzky, G., De Wilde, E., \& Van Heeringen, K. (2008). Deliberate self-harm in young people: Dif- ferences in prevalence and risk factors between The Netherlands and Belgium. European Child \& Adolescent Psychiatry, 17, 179-186. doi:10.1007= s00787-007-0652$\mathrm{X}$

Rizvi, S. L., \& Nock, M. K. (2008). Single-case experimental designs for the evaluation of treatments for self-injurious and suicidal behaviors. Suicide and Life-Threatening Behaviors, 38, 498-510. doi:10.1521=suli.2008.38.5.498

Rodham, K., \& Hawton, K. (2009). Epidemiology and phenomenology of non-suicidal selfinjury. In M. Nock (Ed.), Understanding non-suicidal self- injury: Origins, assessment, and treatment (pp. 37-62). Washington, DC: American Psychological Associ- ation. doi: $10.1037=11875-003$

Ross, S., \& Heath, N. (2002). A study of the frequency of self-mutilation in a community sample of adolescents. Journal Youth Adolescents, 31, 66-77. doi:10.1016=j.psychres.2006.05.010

Ross, S., \& Heath, N. L. (2007). How I Deal With Stress Questionnaire (HIDS). Unpublished manu- script, McGill University, Montreal, Canada.

Sornberger, M. J., Heath, N. L., Toste, J. R., \& McLouth, R. (2012). Non-suicidal self-injury and 
gender: Patterns of prevalence, methods, and loca- tions among adolescents. Suicide and Life-Threatening Behavior, 42, 266-278. doi:10.1111=j.1943-278X. 2012.00088.x

Toste, J. R., Christie, M., \& Heath, N. L. (2011). How I Deal with Stress (HIDS): Evidence for the Use of an NSSI Screening Questionnaire Among Young Adults. Poster session presented at the meeting of the International Society for the Study of Self-Injury, New York, NY.

Toste, J. R., Grouzet, F., Heath, N. L., \& Naeem, A. (2006). A multidimensional exploration of self-injury as a coping strategy. Paper presented at the New England Psychological Association annual meeting, Manchester, $\mathrm{NH}$.

Toste, J. R., Heath, N. L., Grouzet, F., \& Sornberger,

M. J. (2013). Conceptualization of non-suicidal self-injury as a coping strategy: A multidimensional exploration. Manuscript in preparation.

Whitlock, J. L., Powers, J. L., \& Eckenrode, J. (2006). The virtual cutting edge: The Internet and ado- lescent self-injury. Developmental Psychology, 42, 407-417. doi:10.1037=00121649.42.3.407

Williams, F., \& Hasking, P. (2009). Emotion regu- lation, coping, and alcohol use as moderators in the relationship between non-suicidal self-injury and psychological distress. Prevention Science, 11, 33-41. doi:10.1007=s11121-009-0147-8

Yates, T. M., Tracy, A. J., \& Luthar, S. S. (2008). Non-suicidal self-injury among “privileged" youths: Longitudinal and cross-sectional approaches to developmental process. Journal of Consulting and Clinical Psychology, 76, 52-62. doi:10.1037=0022-006X.76.1.52

Zoroglu, S. S., Tuzun, U., Sar, V., Tutkun, H., Savas,

H. A., Ozturk, M., ... Kora, M. E. (2003). Suicide attempt and self-mutilation among Turkish 
This is an Accepted Manuscript of an article published by Taylor \& Francis in 'Archives of Suicide Research' on 2014-07-24, available online: https://www.tandfonline.com/10.1080/13811118.2014.915778.

high school students in relation with abuse, neglect and dissociation. Psychiatry and Clinical Neuros- ciences, 57, 119-126. doi:10.1046=j.1440-1819.2003. 01088.x

\section{Tables and Figures}

TABLE 1. Rates of Lifetime Non-Suicidal Self- Injury (NSSI) Engagement by Participants' Grade

\begin{tabular}{ccc}
\hline & \multicolumn{2}{c}{ Rates Lifetime } \\
\cline { 2 - 3 } & $n$ & $n(\%)$ NSSI \\
\hline Grade 7 & 156 & $46(29.49 \%)$ \\
Grade 8 & 122 & $27(22.13 \%)$ \\
Grade 9 & 352 & $80(22.73 \%)$ \\
Grade 10 & 168 & $35(20.83 \%)$ \\
Grade 11 & 140 & $18(12.86 \%)$ \\
Grade 12 & 153 & $23(15.03 \%)$ \\
\hline
\end{tabular}

FIGURE 1. Frequency of non-suicidal self-injury (NSSI) for those who reported engaging in this behavior. 


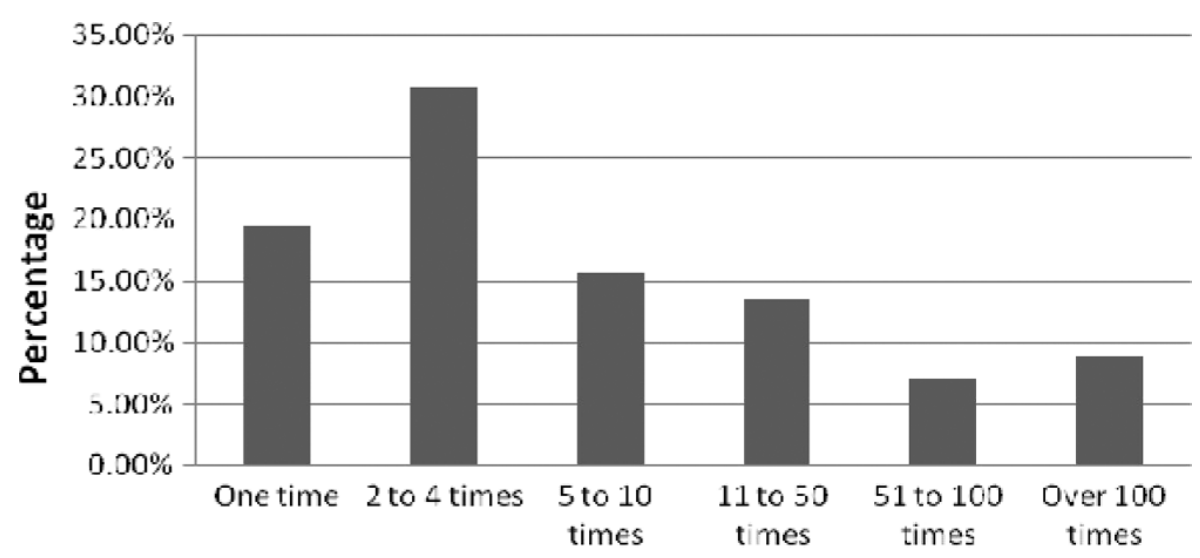

Frequency of NSSI Behavior

FIGURE 2. Age of onset of non-suicidal self-injury (NSSI) for those who reported engaging in this behavior.

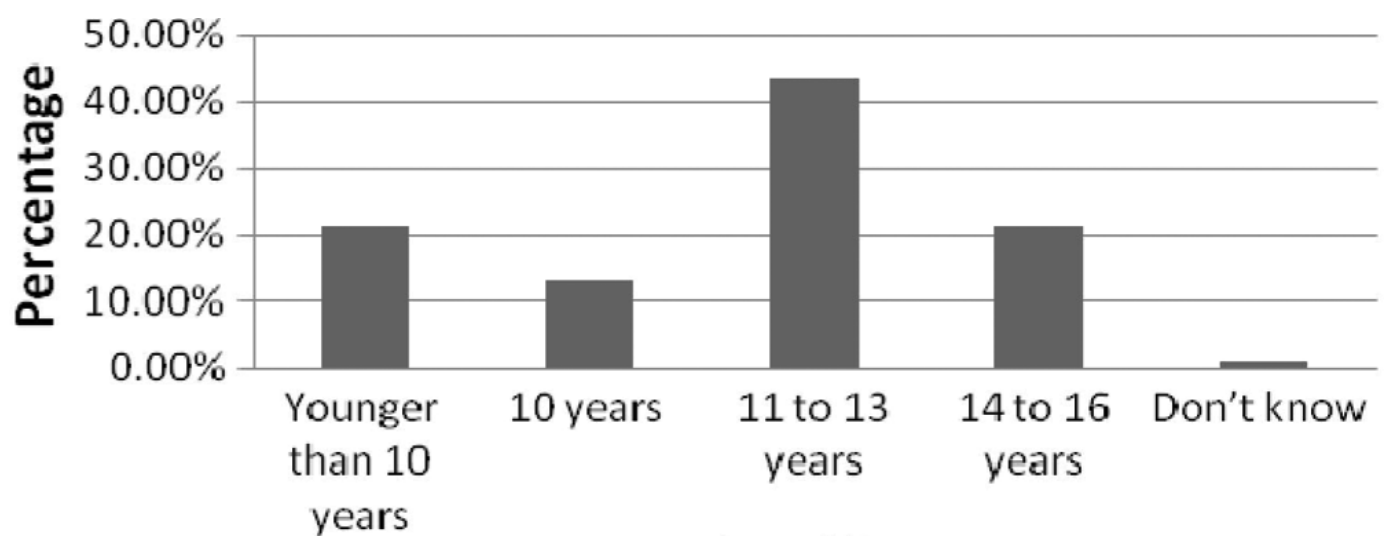

Age of Onset

\begin{tabular}{|l|l|c|c|c|c|}
\hline $\begin{array}{l}\text { TABLE 2. Non-Suicidal Self-Injury (NSSI) Frequency, Age of Onset, and Methods Reported by } \\
\text { Female and Male Adolescents }\end{array}$ \\
\hline \multicolumn{2}{|c|}{ Female } & \multicolumn{2}{c|}{ Male } & \\
\hline Response & $n$ & $\%$ & $n$ & $\%$ & $\mathrm{X}^{2}$ \\
\hline NSSI Frequency & & & & & \\
\hline One Time & 17 & 20.48 & 25 & 20.49 & 0.18 \\
\hline 2 to 4 times & 27 & 32.53 & 39 & 31.97 & 0.001 \\
\hline 5 to 10 times & 15 & 18.07 & 19 & 15.57 & 0.189 \\
\hline 11 to 50 Times & 11 & 13.25 & 18 & 14.75 & 0.111 \\
\hline 51 to 100 Times & 5 & 6.02 & 10 & 8.20 & 0.369 \\
\hline Over 100 times & 8 & 9.64 & 11 & 9.02 & 0.016 \\
\hline Total & 83 & 100 & 122 & 100 & \\
\hline Age of Onset & & & & & \\
\hline Younger than 10 years & 10 & 13.70 & 29 & 26.61 & $4.245^{*}$ \\
\hline
\end{tabular}


This is an Accepted Manuscript of an article published by Taylor \& Francis in 'Archives of Suicide Research' on 2014-07-24, available online: https://www.tandfonline.com/10.1080/13811118.2014.915778.

\begin{tabular}{|l|c|c|c|c|c|}
\hline 10 years & 5 & 6.85 & 19 & 17.43 & $4.221^{*}$ \\
\hline 11 to 13 years & 36 & 49.32 & 42 & 38.53 & 2.294 \\
\hline 14 to 16 years & 21 & 28.77 & 19 & 17.43 & 2.621 \\
\hline Don't know & 1 & 1.37 & 0 & 0.00 & 0.084 \\
\hline Total & 73 & 100 & 109 & 100 & \\
\hline NSSI Methods & & & & & \\
\hline Cutting & 33 & 38.37 & 27 & 22.88 & $6.67^{*}$ \\
\hline Scratching & 38 & 44.19 & 24 & 20.34 & $14.77^{*}$ \\
\hline Punching & 14 & 16.28 & 45 & 38.14 & $10.30^{*}$ \\
\hline Banging head & 23 & 26.74 & 28 & 23.73 & 0.44 \\
\hline Burning & 11 & 12.79 & 9 & 7.63 & 1.75 \\
\hline Other & 19 & 22.09 & 7 & 5.93 & 2.48 \\
\hline Total & 86 & $100 \%$ & 118 & $100 \%$ & \\
\hline Note. $p<.05$. & & & & \\
\hline
\end{tabular}

FIGURE 3. Grade by gender interaction of lifetime rates of non-suicidal self-injury (NSSI) engagement. Note. $p<.01$

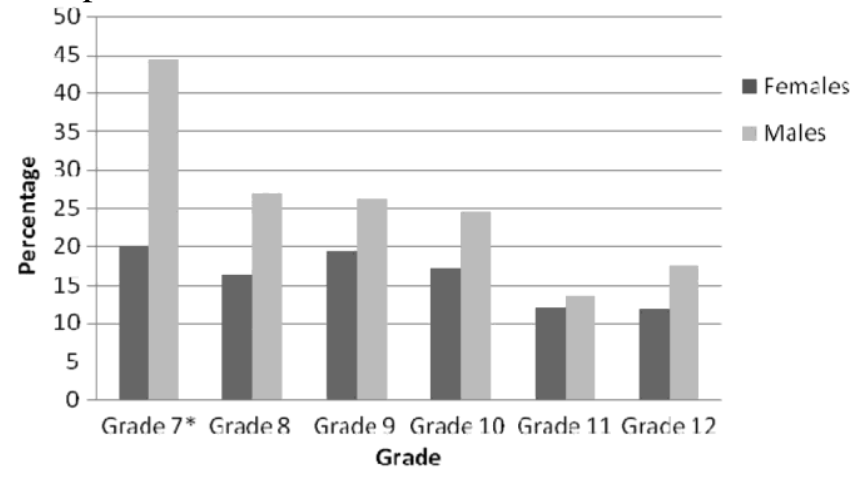

